\title{
Consideraciones de sesgo en la paradoja de la obesidad en pacientes con enfermedad de las arterias coronarias
}

\author{
Bias considerations in the obesity paradox in patients with coronary artery disease
}

Cristian A. Ramos-Vera*

Área de Investigación, Facultad de Ciencias de la Salud, Universidad César Vallejo, Lima, Perú

Al editor:

El reciente artículo de Chambergo-Michilot, et al. ${ }^{1}$ reporta el hallazgo de la hipótesis de la paradoja de la obesidad en mujeres con enfermedad de las arterias coronarias (EAC). Los autores precisan que las pacientes con sobrepeso reportaron menores tasas de mortalidad que aquellas con menor peso.

La paradoja de la obesidad es una hipótesis clínica que refiere que hay mejores pronósticos de salud en las personas obesas que en las personas de peso normal en cuanto a ciertas afecciones médicas ${ }^{2}$, como la EAC. Esto puede tener un impacto controversial, debido a que pone en duda las recomendaciones de reducción de peso en estos pacientes.

La presente carta aclara algunas cuestiones del fenómeno clínico de la paradoja de la obesidad.

El uso de una medida única como el índice de masa corporal (IMC) para evaluar la obesidad es cuestionable. Tal medida no tiene en cuenta directamente la composición corporal, incluyendo la cantidad o la distribución de la grasa, la estructura ósea, la masa muscular y otros cambios asociados con el envejecimiento, lo que puede inducir a un posible hallazgo de la paradoja de la obesidad ${ }^{3}$.

Una de las cuestiones de la investigación de Chambergo-Michilot, et al. ${ }^{1}$ es la pequeña muestra de participantes, conformada mayormente por adultas mayores. En este grupo etario se producen varios cambios fisiopatológicos, como desmineralización ósea, compresión de discos intervertebrales, cifosis dorsal y aplanamiento del arco plantar, entre otros, que provocan una disminución de la medida de la talla que sobrestima el $\mathrm{IMC}^{4}$.

Otro cambio fisiológico debido a la edad, en particular en las mujeres, sucede después de la menopausia: ante la deficiencia de estrógenos se produce un aumento de la cantidad de grasa intermuscular, lo que conlleva una mayor resistencia a la insulina y otra serie de complicaciones, como una alteración desfavorable en las adipocitocinas (elevada proporción de leptina/ adiponectina) y un aumento en la proporción de MMP9/inhibidor a pesar de tener un aceptable IMC ${ }^{5}$.

Las personas obesas no son idénticas y se ven afectadas por su aptitud física personal y su fragilidad, siendo recomendable que la obesidad en adultos se subdivida en las siguientes categorías: clase 1 (IMC de $30-34,9 \mathrm{~kg} / \mathrm{m}^{2}$ ), clase 2 (IMC de $35-39,9 \mathrm{~kg} / \mathrm{m}^{2}$ ) y clase $3\left(\mathrm{IMC}>40 \mathrm{~kg} / \mathrm{m}^{2}\right)^{6}$. Los estudios que no pueden analizar a los participantes obesos en estas categorías no deben asumir la igualdad de subgrupos, debido a que la obesidad no es una variable fija, sino que consta de varias subpoblaciones diferentes ${ }^{6}$. Los participantes de la clase 1 con frecuencia pueden estar más sanos que aquellos con mayor IMC, mientras que los sujetos de clase 3 pueden tener una mayor incidencia de mortalidad por riesgo de alguna enfermedad cardiovascular, como la EAC u otra comorbilidad.

\section{Correspondencia:}

*Cristian A. Ramos-Vera

E-mail: cristony_777@ hotmail.com

0120-5633 / @ 2021 Sociedad Colombiana de Cardiología y Cirugía Cardiovascular. Publicado por Permanyer. Este es un artículo open access bajo la licencia CC BY-NC-ND (http://creativecommons.org/licenses/by-nc-nd/4.0/).

Disponible en internet: 18-10-2021 Rev Colomb Cardiol. 2021;28(4):399-400 www.rccardiologia.com
culo open access bajo la 
La paradoja de la obesidad es un concepto inapropiado $^{7}$, debido a que no hay una teoría plausible que la sostenga ante la falta de fundamentos fisiopatológicos precisos en pacientes con $\mathrm{EAC}^{8}$. Sin embargo, es innegable que la obesidad se asocia con diversas alteraciones metabólicas que aumentan el riesgo de enfermedades cardiovasculares ${ }^{9}$, por ejemplo la EAC en mujeres ${ }^{10}$.

Se requieren evidencias sólidas y concluyentes en futuras investigaciones en pacientes con EAC que evidencien hallazgos de la paradoja de la obesidad para explicar si esta hipótesis es consecuente con verdaderos mecanismos biológicos y no se debe al producto de algún sesgo ${ }^{8}$.

Los futuros estudios deben considerar la influencia de la composición corporal, el estado nutricional y nuevos factores de riesgo, utilizando otras medidas antropométricas adicionales con métodos adecuados para identificar y controlar los posibles sesgos ${ }^{3,8}$. El objetivo es generar una mejor estratificación del riesgo en pacientes con diagnóstico comórbido de obesidad y EAC que permita identificar aquellos con mayor riesgo de mortalidad.

\section{Bibliografía}

1. Chambergo-Michilot D, Runzer-Colmenares FM, Zamora A, Santa-Cruz F, Zamora M. Body mass index and coronary revascularization in women with coronary artery disease: insight into obesity paradox. Rev Colomb Cardiol. 2020:27:380-7.

2. Khan SS, Ning H, Wilkins JT, Allen N, Carnethon M, Berry JD, et al. Association of body mass index with lifetime risk of cardiovascular disease and compression of morbidity. JAMA Cardiol. 2018:3:280-7.

3. Chrysant SG, Chrysant GS. The single use of body mass index for the obesity paradox is misleading and should be used in conjunction with other obesity indices. Postgrad Med. 2019;131:96-102.

4. Gavriilidou NN, Pihlsgård M, Elmståhl S. High degree of BMI misclassification of malnutrition among Swedish elderly population: age-adjusted height estimation using knee height and demispan. Eur J Clin Nutr. 2015;69:565-71.

5. Lizcano F, Guzmán G. Estrogen deficiency and the origin of obesity during menopause. Biomed Res Int. 2014;2014:757461.

6. Zárate A, Basurto AL, Saucedo GRP. La obesidad: conceptos actuales sobre fisiopatogenia y tratamiento. Rev Fac Med UNAM. 2001;44:66-70.

7. Flegal KM, loannidis JPA. The obesity paradox: a misleading term that should be abandoned. Obesity (Silver Spring). 2018;26:629-30.

8. Akin I, Nienaber CA. "Obesity paradox" in coronary artery disease. World J Cardiol. 2015;7:603-8.

9. Prospective Studies Collaboration, Whitlock G, Lewington S, Sherliker P, Clarke R, Emberson J, Halsey J, et al. Body-mass index and cause-specific mortality in 900000 adults: collaborative analyses of 57 prospective studies. Lancet. 2009:373:1083-96.

10. Medina-Inojosa JR, Batsis JA, Supervia M, Somers VK, Thomas RJ, Jenkins $S$, et al. Relation of waist-hip ratio to long-term cardiovascular events in patients with coronary artery disease. Am J Cardiol. 2018;121:903-9. 